\title{
XMM-Newton detection of type I X-ray bursts in M 31 ${ }^{\star}$
}

\author{
W. Pietsch and F. Haberl
}

\author{
Max-Planck-Institut für extraterrestrische Physik, 85741 Garching, Germany \\ e-mail: wnp@mpe.mpg.de
}

Received 2 December 2004 / Accepted 15 December 2004

\begin{abstract}
We searched for X-ray bursts in XMM-Newton archival data of X-ray sources in M 31 globular clusters (GCs) and GC candidates. We detected two bursts simultaneously in EPIC pn and MOS detectors and some more candidates in EPIC pn. The energy distribution of the burst photons and the intrinsic luminosity during the peak of the bursts indicate that at least the strongest burst was a type I radius expansion burst. The bursts identify the sources as neutron star low mass X-ray binaries in M 31. The type I X-ray bursts in M 31 are the first detected outside the Milky Way and show that with the help of XMM-Newton $\mathrm{X}$-ray bursts can be used to classify neutron star low mass X-ray binaries in Local Group galaxies.
\end{abstract}

Key words. galaxies: individual: M 31 - X-rays: galaxies - galaxies: star clusters - X-rays: binaries - X-rays: bursts

\section{Introduction}

Type I X-ray bursts (hereafter X-ray bursts) were detected in low mass X-ray binaries (LMXBs) in the Milky Way and are explained as thermonuclear runaways in freshly accreted material on the surface of a neutron star They may re-occur on a time scale of hours to days, when enough new material is accreted, compressed and heated, to densities and temperatures adequate for another thermonuclear ignition (for reviews see Lewin et al. 1993; Strohmayer \& Bildsten 2003). X-ray bursts show a large variety in profiles and peak flux. The persistent luminosities of the sources when bursting, vary from $\sim 0.2$ to well bellow 0.01 of the Eddington luminosity. Burst rise times last from less than a second to $\sim 10 \mathrm{~s}$, and decay times are in the range of $\sim 10 \mathrm{~s}$ to minutes. In general, burst profiles depend strongly on photon energy, with decays which are much shorter at high photon energies than at low energies. This energy dependence of the burst profile corresponds to a softening of the burst spectrum during decay, which is the result of cooling of the neutron star photosphere. The time dependent energy spectra can be modeled by black body radiation of changing temperature.

During some bursts the energy release is high enough that the luminosity at the surface of the neutron star reaches the Eddington limit leading to an expansion of the neutron star atmosphere (radius expansion X-ray bursts). These bursts show an energy dependent double peak structure. During expansion and subsequent contraction the luminosity remains almost constant near the Eddington limit. However, the black body temperature may quickly drop from more than $3 \mathrm{keV}$ to well below $0.5 \mathrm{keV}$ when the radius of the photosphere expands before

^ XMM-Newton is an ESA Science Mission with instruments and contributions directly funded by ESA Member States and the USA (NASA). it slowly rises again during contraction to $2-3 \mathrm{keV}$ and then cools thereafter with continuously decreasing luminosity. For a detailed description of the temperature and flux development of several radius expansion bursts from 4U/MXB 1820-30 see Haberl et al. (1987). The radius expansion bursts of about two thirds of the sources reach a critical bolometric luminosity of $\sim 3.8 \times 10^{38} \mathrm{erg} \mathrm{s}^{-1}$, corresponding to the Eddington limit for hydrogen-poor matter and may be used as standard candles (see e.g. Kuulkers et al. 2003). The expansion phase may last for up to $30 \mathrm{~s}$. To date thirteen luminous globular cluster (GC) $\mathrm{X}$-ray sources are known in the Milky Way, twelve of which show X-ray bursts (and sometimes even radius expansion X-ray bursts) and therefore are believed to be LMXBs containing a neutron star (see e.g. Verbunt \& Lewin 2004). The distances of the sources vary between about 4 and $12 \mathrm{kpc}$. Up to now, no $\mathrm{X}$-ray bursts have been detected outside the Milky Way.

With the high collecting power and good position resolution of the XMM-Newton EPIC cameras we now have the possibility to detect X-ray burst sources in all galaxies of the Local Group. The X-ray burst signature then will identify these sources as neutron star LMXBs. Using the maximum burst flux as standard candle would allow to determine the distance of the burst source. However, due to the limiting statistics, distance determinations by this method can not compete with the accuracy of other methods. The persistent luminosity of many bursting LMXBs may be below the limiting point source sensitivity of current observations. Therefore, to find all X-ray burst sources in neighboring galaxies, one would have to search the entire extent of the galaxy with the resolution of the instrument point spread function (PSF). A less complete method would be a search in LMXB candidates selected by other means (e.g. as GC X-ray sources). Radius expansion bursts would be most easily picked up as they are the most luminous "standard" 
type I X-ray bursts. Besides these bursts one may detect type I "superbursts" lasting for several hours with peak luminosities above $10^{38} \mathrm{erg} \mathrm{s}^{-1}$ and maximum black body temperatures of 2-3 keV similar to the ones detected with BeppoSAX and RXTE from LMXBs in the Milky Way (see e.g. Strohmayer \& Bildsten 2003).

An ideal target for a search for X-ray burst sources is the bright Local Group spiral M 31 (distance 780 kpc, Holland 1998; Stanek \& Garnavich 1998) with its moderate Galactic foreground absorption $\left(N_{\mathrm{H}}=7 \times 10^{20} \mathrm{~cm}^{-2}\right.$, Stark et al. 1992). Many GC and GC candidates are known in M 31 (see e.g. Galleti et al. 2004) which may host LMXBs. Pietsch et al. (2005, hereafter PFH2005) prepared a catalogue of M 31 point-like X-ray sources analyzing all observations in the XMM-Newton archive which overlap at least in part with the optical $D_{25}$ extent of the galaxy. In total, they detected 856 sources and identified and classified them using their X-ray properties and correlations with sources in other wavelength regimes. Twenty-seven of the X-ray sources are identified with GCs and ten with GC candidates. They are - extrapolating from bright GC sources in the Milky Way - most likely neutron star LMXBs. In this paper we report on a search for X-ray bursts in the M 31 XMM-Newton data of these LMXB candidates.

\section{XMM-Newton observations and analysis}

We searched for M 31 burst sources in the same archival XMM-Newton (Jansen et al. 2001) EPIC (Strüder et al. 2001; Turner et al. 2001) observations that were used by PFH2005 for the creation of the $M 31$ source catalogue, i.e. pointings $\mathrm{c} 1$ to $\mathrm{c} 4$ to the galaxy centre, $\mathrm{n} 1$ to $\mathrm{n} 3$ to the northern disk, $\mathrm{s} 1$ and $\mathrm{s} 2$ to the southern disk and h4 to the northwest halo (see Table 1 of PFH2005, for details). The observations were performed in the full frame mode (time resolution for pn and MOS $73.4 \mathrm{~ms}$ and $2.6 \mathrm{~s}$, respectively) using medium or thin filter with low background exposure times of about 10 to $50 \mathrm{ks}$. For the light curve analysis we used event pattern up to 12 (singles to quadruples). We restricted our search to the stringent low background times used by PFH2005 to avoid spurious burst candidates due to background flares which would show up in all EPIC instruments at the same time and might be misidentified as bursts.

To get an estimate for the counts that can be expected in the EPIC detectors from an X-ray burst in M 31, we calculated count rates for typical burst spectra (black body spectra with temperatures of 0.1 to $3 \mathrm{keV}$ ) for a source with an intrinsic luminosity of $10^{38} \mathrm{erg} \mathrm{s}^{-1}$ (Table 1). It is clear that only bursts with radius expansion, that radiate at the Eddington luminosity for at least several seconds will give enough photons (more than about 10) to be significantly detected in pn detector and possibly also several photons for burst confirmation in the MOS detectors. The expected number of photons in the individual MOS instruments is lower than for the pn by a factor of two or more depending on the assumed spectrum (see Table 1). The drop of the black body temperature during the expansion to values below $1 \mathrm{keV}$ additionally favors their detection as it shifts the main energy output into the band where EPIC is most sensitive. Taking all the points mentioned above into account, a search strategy for bursts should be based on pn light curves of
Table 1. XMM-Newton EPIC count rates for the pn and one MOS instrument (thin filter full frame mode, 15 arcsec on-axis extraction radius corresponding to approximately the half energy width of the PSF) in different energy bands (in $\mathrm{keV}$ ) expected from a source emitting a black body spectrum with temperature $T_{\mathrm{bb}}$ and intrinsic bolometric luminosity of $10^{38} \mathrm{erg} \mathrm{s}^{-1}$ at the M 31 distance assuming Galactic foreground absorption of $7 \times 10^{20} \mathrm{~cm}^{-2}$.

\begin{tabular}{rrrrrrr}
\hline \hline \multicolumn{1}{c}{$T_{\mathrm{bb}}$} & \multicolumn{3}{c}{ EPIC pn } & \multicolumn{5}{c}{ EPIC MOS } \\
& $0.2-0.5$ & $0.5-4.5$ & $4.5-7.0$ & $0.2-0.5$ & $0.5-4.5$ & $4.5-7.0$ \\
$(\mathrm{keV})$ & & \multicolumn{5}{c}{$\left(10^{-3}\right.$ counts s $\left.^{-1}\right)$} \\
\hline 0.1 & 124 & 125 & 0 & 23 & 30 & 0 \\
0.2 & 70 & 364 & 0 & 14 & 109 & 0 \\
0.5 & 10 & 333 & 2 & 2 & 126 & 1 \\
0.8 & 3 & 213 & 13 & 1 & 85 & 5 \\
1.0 & 2 & 158 & 21 & 0 & 65 & 8 \\
1.5 & 0 & 79 & 26 & 0 & 33 & 9 \\
2.0 & 0 & 44 & 22 & 0 & 19 & 8 \\
3.0 & 0 & 17 & 12 & 0 & 7 & 4 \\
\hline
\end{tabular}

candidate sources with time resolutions of about $10 \mathrm{~s}$ or longer and use MOS count rates for confirmation.

Therefore, we created light curves for EPIC pn in the $0.5-4.5 \mathrm{keV}$ band with $500 \mathrm{~s}, 100 \mathrm{~s}$ and $20 \mathrm{~s}$ resolution for the 37 sources associated with a GC or possibly associated with a GC in the catalogue of PFH2005 selecting all events within a radius around the source corresponding to the half energy width of the PSF at the off-axis angle. We rejected light curves when the source was outside the EPIC pn field of view (FOV) or partly covered by EPIC pn CCD gaps. The effective integrated exposure time for all sources adds up to $2.88 \times 10^{6} \mathrm{~s}$. We inspected the light curves by eye for burst candidates. Two bursts are confirmed in MOS light curves and are therefore regarded as secure detections. Background flares are ruled out as they would appear simultaneously in other source light curves. The bursts most likely showed radius expansion and will be discussed in greater detail in the following sections. Several other pn burst candidates were not in the FOV of the MOS detectors or not detected by them. No burst with characteristics similar to the superbursts in the Galaxy could be detected. However, as also in the Galaxy superbursts are very rare events, it is not surprising that none appears in the available M 31 observations.

The data analysis was performed using tools in the XMM-Newton Science Analysis System (SAS) v6.0.0, EXSAS/MIDAS 03OCT_EXP, and FTOOLS v5.2 software packages, the imaging application DS9 v3.0b6 together with the funtools package, the mission count rate simulator WebPIMMS v3.6a and the spectral analysis software XSPEC v11.3.1.

\section{The X-ray burst source in the globular cluster [WSB85] S5 15}

The brightest M 31 burst in the EPIC pn and both MOS instruments was detected from source [PFH2005] 253 in GC [WSB85] S5 15 (Wirth et al. 1985) during observation 


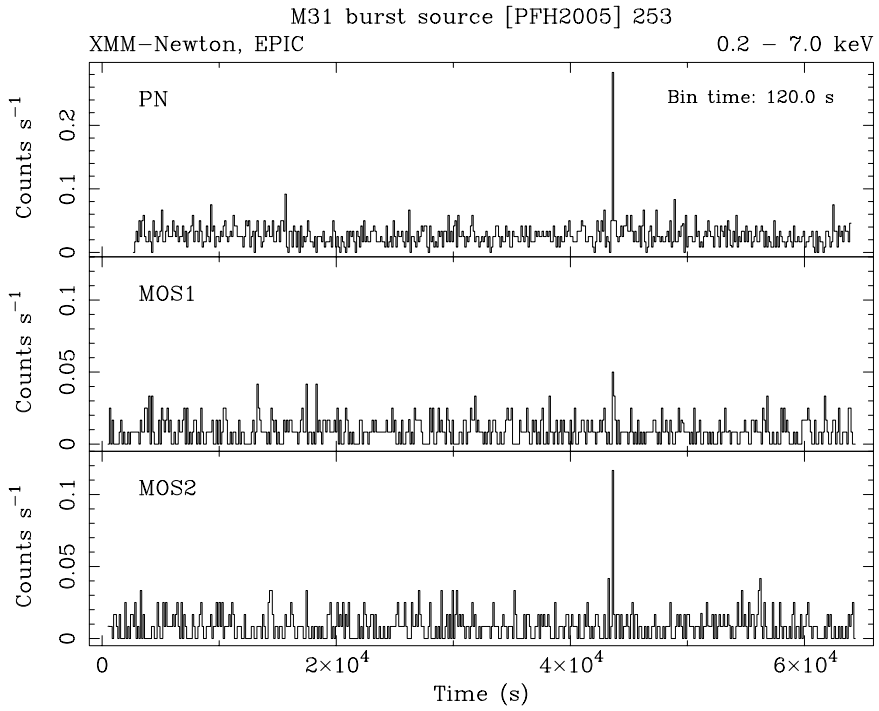

Fig. 1. XMM-Newton EPIC light curves of source [PFH2005] 253 during the M 31 centre observation c4 (OBSID 0112570101) on January 6/7, 2002 integrated over $120 \mathrm{~s}$. Time zero corresponds to the start of the observation.

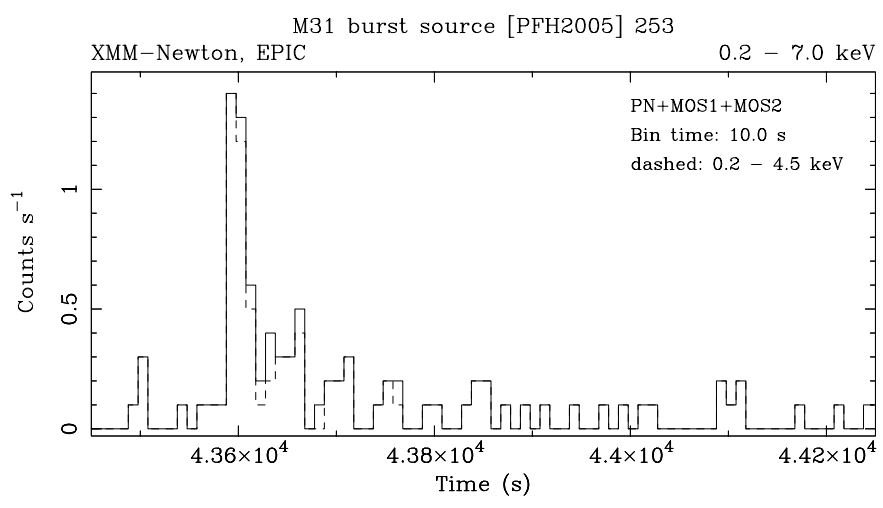

Fig. 2. Combined XMM-Newton EPIC light curve (pn, MOS1 and MOS2 added) of the burst in Fig. 1 of source [PFH2005] 253 integrated over $10 \mathrm{~s}$. The dashed histogram shows the light curve of the $0.2-4.5 \mathrm{keV}$ events.

c4 starting at UT 6:14:50 on January 7, 2002 (Figs. 1 and 2). It reached its maximum luminosity within $10 \mathrm{~s}$, stayed at the maximum (count rate in $0.2-7 \mathrm{keV}$ band of $(1.4 \pm 0.4) \mathrm{ct} \mathrm{s}^{-1}$ ) for less than $20 \mathrm{~s}$ and decayed to background level within about $150 \mathrm{~s}$. Due to limiting statistics the structure of the bursts can not be further resolved. In total, more than 50 burst counts are detected. As can be seen from Fig. 2 photons in the 4.5-7 keV band are only detected after the maximum of the burst as expected for the temperature development in a radius expansion burst. Also the maximum count rate corresponds to that of a M 31 source with a $1 \mathrm{keV}$ black body spectrum radiating at $3.8 \times 10^{38} \mathrm{erg} \mathrm{s}^{-1}$, i.e. at the Eddington limit for hydrogen-poor matter.

Source [PFH2005] 253 was in the EPIC FOV during the four XMM-Newton M 31 centre pointings c1 to c4 (average absorbed luminosity of $3.4 \times 10^{36} \mathrm{erg} \mathrm{s}^{-1}$ in $0.2-4.5 \mathrm{keV}$ band). Between the pointings which are equally spread over two years, its average luminosity varied by a factor of $\sim 6$. During pointing

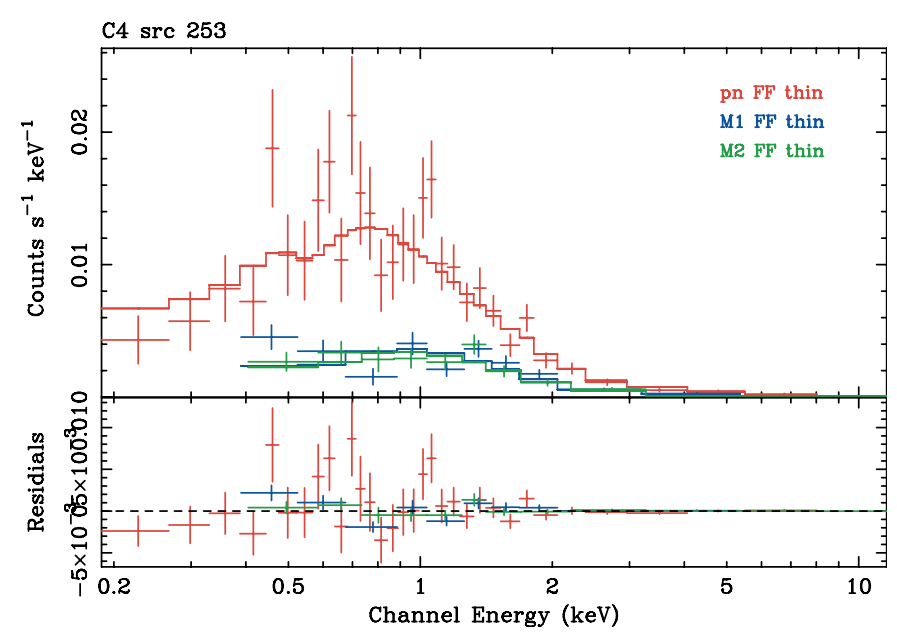

Fig. 3. XMM-Newton EPIC spectrum of source [PFH2005] 253 for observation c4. The best fit to the three EPIC spectra - absorbed bremsstrahlung plus black body model - is shown in the upper panel ( $\mathrm{pn}$ is the upper spectrum and the two MOS spectra overlap).

c1 [PFH2005] 253 was partly hidden in pn by a CCD gap. We therefore only used pointings $\mathrm{c} 2, \mathrm{c} 3$ and $\mathrm{c} 4$ for the burst search (effective low background time of $75.7 \mathrm{ks}$ ).

During observation c4, more than 2500 photons were detected in the EPIC detectors from [PFH2005] 253. An absorbed bremsstrahlung plus black body model describes the persistent energy spectrum (see Fig. 3, best fitting model parameters: $\quad N_{\mathrm{H}}=\left(9_{-5}^{+13}\right) \times 10^{20} \mathrm{~cm}^{-2}, \quad T_{\text {brems }}=5_{-2}^{+20} \mathrm{keV}$, $T_{\mathrm{bb}}=0.18_{-0.08}^{+0.06} \mathrm{keV}$, reduced $\chi_{\text {min }}^{2}=1.66$ for 45 degrees of freedom) and corresponds to an absorbed luminosity and intrinsic luminosity of the source in the $0.2-4.5 \mathrm{keV}$ band of 4.1 and $5.6 \times 10^{36} \mathrm{erg} \mathrm{s}^{-1}$, respectively. The source was also detected by Chandra ACIS-I and HRC as a variable source (r3-41, Kong et al. 2002; CXOM31 J004221.5+411419, Kaaret 2002).

\section{The X-ray burst source in the globular cluster B150}

The EPIC pn and the MOS2 instruments detected a burst from M 31 source [PFH2005] 396 in GC B150 (Galleti et al. 2004) during observation c3 starting at UT 16:58:00 on June 29, 2001 (Fig. 4). In MOS1 the source was heavily obscured by a CCD gap and therefore MOS1 data could not be used for the analysis. The burst was significantly fainter (about a factor of three) than the burst of [PFH2005] 253 and only about 15 counts are detected, four or them in MOS2. The burst lasted for about $40 \mathrm{~s}$ with a rise to maximum within $10 \mathrm{~s}$. Due to limiting statistics the structure of the bursts can not be further resolved. The count rate at maximum provides us with a lower limit to the peak luminosity in M 31 (assuming a $1 \mathrm{keV}$ black body spectrum) of about $10^{38} \mathrm{erg} \mathrm{s}^{-1}$.

Source [PFH2005] 396 was in the EPIC FOV during the four XMM-Newton M 31 centre pointings c1 to c4 (average absorbed luminosity of $1.7 \times 10^{36} \mathrm{erg} \mathrm{s}^{-1}$ in $0.2-4.5 \mathrm{keV}$ band). Between the pointings, its absorbed luminosity varied by a 

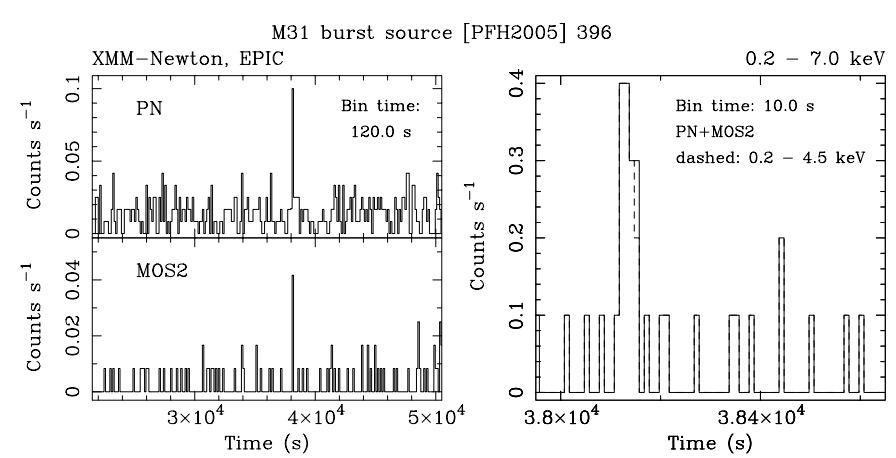

Fig. 4. Source [PFH2005] 396: XMM-Newton EPIC pn and MOS2 light curves during the M 31 centre observation c3 (OBSID 0112570701) on June 29, 2001 integrated over $120 \mathrm{~s}$ (left). Combined XMM-Newton EPIC light curve (pn and MOS2 added) of the burst integrated over $10 \mathrm{~s}$ (right). Time zero corresponds to the start of the observation.

factor of $\sim 1.7$. We used pointings $\mathrm{c} 1, \mathrm{c} 2, \mathrm{c} 3$ and $\mathrm{c} 4$ for the burst search (effective low background time of $99.3 \mathrm{ks}$ ).

During observation $\mathrm{c} 3$, the source was at a luminosity of $(2.0 \pm 0.2) \times 10^{36} \mathrm{erg} \mathrm{s}^{-1}$. The faintness of the source and the low background exposure time for observation $\mathrm{c} 3$ that was a factor of two shorter than for observation c4, prevented a similar spectral modeling as for [PFH2005] 253. The source was also detected by Chandra ACIS-I and HRC as a variable source (r3-18, Kong et al. 2002; CXOM31 J004307.4+412021, Kaaret 2002).

\section{Discussion}

We have detected the first type I X-ray bursts outside the Milky Way from two M 31 GC source candidates of PFH2005 which showed persistent luminosities while bursting of below $\sim 5 \times 10^{36} \mathrm{erg} \mathrm{s}^{-1}$. In search for bursts we checked low background EPIC pn light curves (covering in total $2.88 \times 10^{6} \mathrm{~s}$ ) of sources associated with GCs or possibly associated with GCs. The persistent luminosities of part of the sources was above 0.2 times the Eddington luminosity of a neutron star and therefore, extrapolating from the experience of Galactic LMXBs, were not expected to show bursts during these bright states. No burst with characteristics similar to the superbursts in the Galaxy was detected.

The peak luminosities of the two bursts in [WSB85] S5 15 and $\mathrm{B} 150$ are comparable to those of bright radius expansion bursts in the Milky Way. The detections demonstrate the possibility to classify neutron star LMXBs in the Local
Group by their bursting signature using just X-ray information from XMM-Newton EPIC. The energy response of the EPIC instruments is most sensitive to detect radiation with black body temperatures in the range $0.2-1 \mathrm{keV}$ which are normally covered for some time during the contraction phase of the radius expansion when the burst is radiating at the Eddington luminosity. Of specific importance is the possibility to simultaneously detect a burst in more than one EPIC instrument, which gives high confidence in the credibility of a burst even if only few photons are involved. In this work only known LMXB candidates in M 31 from PFH2005 were investigated. The success encourages a more systematic survey covering the total area of M 31 and also other Local Group galaxies to identify neutron star LMXBs just from their bursting properties. However, to be able to use the bursts as standard candles as proposed by Kuulkers et al. (2003) even better statistics at higher time resolution are needed to analyze the burst signature in more detail.

Acknowledgements. The XMM-Newton project is supported by the Bundesministerium für Bildung und Forschung / Deutsches Zentrum für Luft- und Raumfahrt (BMBF/DLR), the Max-Planck Society and the Heidenhain-Stiftung.

\section{References}

Galleti, S., Federici, L., Bellazzini, M., Fusi Pecci, F., \& Macrina, S. 2004, A\&A, 416, 917

Haberl, F., Stella, L., White, N. E., Gottwald, M., \& Priedhorsky, W. C. 1987, ApJ, 314, 266

Holland, S. 1998, AJ, 115, 1916

Jansen, F., Lumb, D., Altieri, B., et al. 2001, A\&A, 365, L1

Kaaret, P. 2002, ApJ, 578, 114

Kong, A. K. H., Garcia, M. R., Primini, F. A., et al. 2002, ApJ, 577, 738

Kuulkers, E., den Hartog, P. R., in't Zand, J. J. M., et al. 2003, A\&A, 399,663

Lewin, W. H. G., van Paradijs, J., \& Taam, R. E. 1993, Space Sci. Rev., 62, 223

Pietsch, W., Freyberg, M., \& Haberl, F. 2005, A\&A, in press [arXiv: astro-ph/0410117] (PFH2005)

Stanek, K. Z., \& Garnavich, P. M. 1998, ApJ, 503, L131

Stark, A. A., Gammie, C. F., Wilson, R. W., et al. 1992, ApJS, 79, 77

Strüder, L., Briel, U., Dennerl, K., et al. 2001, A\&A, 365, L18

Strohmayer, T., \& Bildsten, L. 2003, in Compact Stellar X-ray Sources, in press [arXiv: astro-ph/0301544]

Turner, M. J. L., Abbey, A., Arnaud, M., et al. 2001, A\&A, 365, L27

Verbunt, F., \& Lewin, W. H. G. 2004, in Compact Stellar X-ray Sources, in press [arXiv: astro-ph/0404136]

Wirth, A., Smarr, L. L., \& Bruno, T. L. 1985, ApJ, 290, 140 